\title{
Antioxidant-mediated protective role of Hericium erinaceus (Bull.: Fr.) Pers. against oxidative damage in fibroblasts from Friedreich's ataxia patient
}

\author{
Sze-Yuen LEW ${ }^{1}$, Yoon-Yen YOW², Lee-Wei LIM³ ${ }^{3}$ Kah-Hui WONG ${ }^{1 *}$ (D)
}

\begin{abstract}
Friedreich's ataxia (FRDA) is a progressive neuromuscular disorder caused by substantial decrease of mitochondrial protein frataxin responsible for biogenesis of iron-sulphur clusters and protection from oxidative damage. In this study, we investigated the antioxidant activities of a standardized aqueous extract from fruiting bodies of Hericium erinaceus mushroom (HESAE) and its protective effects against oxidative damage induced by L-Buthionine sulfoximine (BSO) in fibroblasts derived from FRDA patient. The lactate dehydrogenase-based viability assay showed that FRDA fibroblast was sensitive to $12.5 \mathrm{mM}$ BSO with a reduction of viability to $52.51 \pm 13.92 \%$ after $24 \mathrm{~h}$ of BSO exposure. Interestingly, co-incubation with $32 \mathrm{mg} / \mathrm{mL}$ HESAE increased the viability to $85.35 \pm 3.4 \%$. Further, $12.5 \mathrm{mM}$ BSO caused a decrease in the ratio of cellular reduced glutathione (GSH) to oxidised GSH (GSSG) that leads to cell death. Nevertheless, the damage was reduced by co-incubation with $32 \mathrm{mg} / \mathrm{mL}$ HESAE. Nuclear fluorescence staining revealed that $12.5 \mathrm{mM} \mathrm{BSO}$ induced cell death and the apoptosis was decreased by co-incubation with HESAE. These findings suggest the ability of HESAE in attenuating BSO-mediated cytotoxicity through maintenance of membrane integrity and optimal GSH/GSSG ratio, that are closely linked to its antioxidant activities. Further in vivo trials are highly warranted to clarify its potential benefits in management of FRDA.
\end{abstract}

Keywords: Hericium erinaceus; Friedreich's ataxia; dermal fibroblasts; antioxidant; oxidative damage.

Practical Application: A standardized aqueous extract of Hericium erinaceus is beneficial in attenuating BSO-mediated oxidative damage in fibroblasts from Friedreich's ataxia patient. The medicinal mushroom acts as a potent protector against oxidative damage-induced cell death and could be a possible therapeutic for delaying the FRDA symptoms. The observed effects were better than idebenone, a lipid antioxidant that has mild adverse effects. Further, the cellular model is an important tool for the discovery of novel therapeutic approach in FRDA in which reactive radicals and oxidative damage are involved.

\section{Introduction}

In the 1860s, Nikolaus Friedreich, a German neurologist described a mysterious inherited disease marked by progressive loss of coordination and neuronal degeneration, and coined the term Friedreich's ataxia (FRDA). The prevalence of the most common hereditary ataxia was estimated at 1 in 29000 , with a carrier frequency of 1 in 85 in the individuals of Caucasian descent (Delatycki \& Corben, 2012; Koeppen, 2013). FRDA is known to be a debilitating, life-shortening and degenerative neuromuscular disorder where the patients suffer from progressive gait and limb ataxia, lack of tendon reflexes in the legs, dysarthria, slurred speech and pyramidal weakness of lower limbs. The non-neurological symptoms include hypertrophic cardiomyopathy, scoliosis, diabetes mellitus or skeletal deformities (Delatycki \& Corben, 2012).

The pathogenic mutation in FRDA is caused by expansion of guanine-adenine-adenine (GAA) trinucleotide repeat in the first intron of the frataxin gene on chromosome 9q13-21 that leads to a substantial decrease of mitochondrial protein frataxin

(Jauslin et al., 2007). Reduced frataxin level causes an increase of cellular oxidative damage and impaired formation of iron-sulfur (Fe-S) clusters such as heme, electron transport chain (ETC) complexes I-III and the Kreb's cycle protein aconitase (Lodi et al., 2006).

In recent years, culinary and medicinal mushrooms are gaining considerable attention for potentials in promoting neuronal health. One such mushroom, Hericium erinaceus (Bull.:Fr.) Pers. or lion's mane mushroom is increasingly studied for its neuroprotective effects. It is one of the culinary and medicinal mushrooms found in Asia, Europe and North America (Wong et al., 2011).

Hericium erinaceus has been extensively tested in in vitro trials as the neurite outgrowth stimulator in the cultured cells of neural hybrid clone NG108-15 and rat pheochromocytoma (Wong et al., 2007). We also explored its ability in the enhancement of peripheral nerve regeneration and acceleration of motor and sensory functional recovery after crush injury (Wong et al., 2011, 
2015). Inanaga (2014) reported an improved neurocognitive function in an 86-year-old male patient with recurrent depressive disorder while Okamura et al. (2015) reported improved sleep quality and subjective well-being among female undergraduate students after taking the mushroom tablets, Amyloban ${ }^{\circledR} 3399$. In a study by Nagano et al. (2010), H. erinaceus cookies was shown to reduce depression and anxiety in 30 females between age 40-45. The broad therapeutic benefits of $H$. erinaceus may be associated to its strong antioxidant effects (Friedman, 2015). Therefore, the potential of $H$. erinaceus in combating oxidative damage-related neurodegenerative diseases including hereditary ataxia could be explained by the neuroprotective activity and its plentiful source of exogenous antioxidants.

This prompted our interest to investigate the protective effects of a standardized aqueous extract from fruiting bodies of $H$. erinaceus (HESAE) against oxidative damage induced by L-Buthionine sulfoximine (BSO) in fibroblasts from a FRDA patient and to link the effectiveness to its antioxidant properties. To the best of our knowledge, there are no reports on therapeutic application of medicinal mushrooms in FRDA. HESAE is a dietary supplement manufactured in Malaysia that targets on the general health maintenance. BSO is a specific inhibitor of $\gamma$-glutamylcysteine synthetase and its downregulation results in reduction of mitochondrial glutathione, increased oxidative damage and decreased mitochondrial function (Reliene \& Schiestl, 2006).

\section{Materials and methods}

\subsection{Mushroom sample}

The aqueous extract of $H$. erinaceus (HESAE; $\mathrm{NevGro}^{\circledR}$; batch No. 7H2308X) was obtained from Ganofarm R\&D Private Limited, Tanjung Sepat, Selangor, Malaysia. $\mathrm{NevGro}^{\circledR}$ is a standardized aqueous extract from fresh fruiting bodies of $\mathrm{H}$. erinaceus that contains not less than $20.66 \%$ beta 1,3-1,6 glucan and $0.17 \%$ adenosine (Nova Laboratories Private Limited, Sepang, Selangor, Malaysia). Total glucan and $\alpha$-glucan were determined by the $\beta$-glucan assay kit (yeast \& mushroom) K-YBGL (Megazyme International, Wicklow, Ireland). The $\beta$-glucan content was calculated by subtracting the $\alpha$-glucan from the total glucan content. Adenosine content was analyzed and quantified by high-performance liquid chromatography (HPLC) using an in-house method (Nova Laboratories Private Limited, Sepang, Selangor, Malaysia). A stock solution of $64 \mathrm{mg} / \mathrm{mL}$ was prepared by dissolving HESAE in distilled water for the assessment of phytochemical contents and in vitro antioxidant activities or in phenol red-free Dulbecco's Modified Eagle Medium (DMEM) and filter sterilized through a $0.2 \mu \mathrm{m}$ nylon membrane filter for the measurement of oxidative damage.

\subsection{Determination of total phenolic content}

The concentration of phenolic compounds in HESAE and idebenone (Cayman Chemical, Ann Arbor, MI, USA), expressed as gallic acid equivalent (GAE), was determined according to a method as described by Singleton et al. (1999) and Pang et al. (2018).

\subsection{Determination of total flavonoid content}

The concentration of flavonoid compounds in HESAE and idebenone, expressed as quercetin equivalent $(\mathrm{QE})$, was determined according to a method as described by Pękal \& Pyrzynska (2014) and Pang et al. (2018).

\subsection{DPPH free radical scavenging assay}

The scavenging activity of HESAE on 2,2-diphenyl-1picrylhydrazyl (DPPH) radical was measured according to the method of Brand-Williams et al. (1995). Idebenone was used as a positive control. The scavenging ability of HESAE was expressed as $\mathrm{EC}_{50}$ value $(\mathrm{mg} / \mathrm{mL})$, which is the effective concentration at which $50 \%$ of DPPH radicals were scavenged.

\subsection{ABTS free radical scavenging assay}

The scavenging activity of HESAE on 2,2'-azinobis(3-ethylbenzothiazoline-6-sulfonic acid) (ABTS) radical was measured according to the method of Miller et al. (1993). Idebenone was used as a positive control. The scavenging ability of HESAE was expressed as $\mathrm{EC}_{50}$ value $(\mathrm{mg} / \mathrm{mL})$, which is the effective concentration at which $50 \%$ of ABTS radicals were scavenged.

\subsection{Reducing power assay}

Reducing power of HESAE was determined according to the method of Oyaizu (1986). Idebenone was used as a positive control. The reducing power of HESAE was expressed as $\mathrm{EC}_{50}$ value $(\mathrm{mg} / \mathrm{mL})$, at which the absorbance was 0.5 .

\subsection{Dermal fibroblasts culture}

Dermal fibroblasts from a 30-year-old FRDA male patient (GM04078) and dermal fibroblasts from a healthy 11-year-old female (GM02036) were purchased from Coriell Institute (Camden, NJ, USA). The fibroblasts were maintained in Dulbecco's Modified Eagle Medium (DMEM) supplemented with $15 \%$ fetal bovine serum and $1 \%$ penicillin-streptomycin at $37 \pm 2{ }^{\circ} \mathrm{C}$ in a $5 \%$ $\mathrm{CO}_{2}$-humidified incubator. Prior to L-Buthionine sulfoximine (BSO), BSO-HESAE or BSO-idebenone treatment, the medium was changed to phenol red- and sodium pyruvate-free DMEM containing $1 \%$ fetal bovine serum and $1 \%$ penicillin-streptomycin. Fibroblasts treated with $5 \mu \mathrm{M}$ of idebenone served as a positive control (Jauslin et al., 2002), while fibroblasts in complete DMEM without treatment served as a negative control.

\subsection{3-(4,5-dimethylthiazol-2-yl)-2,5-diphenyltetrazolium bromide (MTT) viability assay}

MTT assay was used to assess the effect of BSO or HESAE on viability of normal and FRDA fibroblasts. Fibroblasts were plated at a density of $5 \times 10^{3}$ cells per well in 96-well plates and incubated for $24 \mathrm{~h}$ at $37 \pm 2{ }^{\circ} \mathrm{C}$ in a $5 \% \mathrm{CO}_{2}$-humidified incubator. Then, the medium was replaced with fresh medium containing BSO or HESAE. After $24 \mathrm{~h}$ of incubation, the morphology of fibroblasts was acquired on Nikon Eclipse Ti-S inverted microscope equipped with a digital camera controller (DS-U3) and NIS-Elements algorithm (NIS-Elements Advanced Research, 
Nikon Corporation, Tokyo, Japan). The extent of MTT reduction was determined by measuring the absorbance at $570 \mathrm{~nm}$ with $630 \mathrm{~nm}$ as background absorbance in a multimode microplate reader (SpectraMax M3, Molecular Devices, San Jose, CA, USA). The fibroblasts in complete DMEM without treatment served as a negative control. The $50 \%$ inhibitory concentration $\left(\mathrm{IC}_{50}\right)$ was interpolated from the response curve.

\subsection{Lactate dehydrogenase (LDH) viability assay}

$\mathrm{LDH}$ assay was used to determine the protective effect of HESAE against BSO-induced cytotoxicity in FRDA fibroblasts based on the measurement of LDH released from the cytosol of damaged fibroblasts into the supernatant. The fibroblasts were plated at a density of $5 \times 10^{3}$ cells per well in 96-well plates and incubated for $24 \mathrm{~h}$ at $37 \pm 2{ }^{\circ} \mathrm{C}$ in a $5 \% \mathrm{CO}_{2}$-humidified incubator. The medium was replaced with fresh medium containing BSO, BSO-HESAE or BSO-idebenone and incubated for another $24 \mathrm{hr}$. Fibroblasts treated with $5 \mu \mathrm{M}$ of idebenone served as a positive control while fibroblasts in complete DMEM without treatment served as a negative control. After incubation, the plate was centrifuged at $1500 \mathrm{rpm}$ for $5 \mathrm{~min}$ at $18{ }^{\circ} \mathrm{C}$. Culture supernatant was subjected to $\mathrm{LDH}$ measurement according to the manufacturer's protocol of LDH cytotoxicity detection kit (Roche, Mannheim, Germany). LDH activity was determined by measuring the absorbance at $492 \mathrm{~nm}$ with $690 \mathrm{~nm}$ as background absorbance in a multimode microplate reader (SpectraMax M3, Molecular Devices, San Jose, CA, USA).

\subsection{Reduced glutathione/oxidized glutathione (GSH/GSSG) assay}

GSH/GSSG assay is a luminescence-based system for the detection and quantification of total glutathione and oxidised glutathione (GSSG) in cultured cells. The ratio of reduced glutathione (GSH) to oxidised GSH (GSSG) is an indicator of cellular health, with reduced GSH making up to $98 \%$ of cellular GSH under normal conditions. Fibroblasts were plated at a density of $5 \times 10^{3}$ cells per well in white opaque 96 -well plates and incubated for $24 \mathrm{~h}$ at $37^{\circ} \mathrm{C}$ in a $5 \% \mathrm{CO}_{2}$-humidified incubator. The medium was replaced with fresh medium containing BSO, BSO-HESAE or BSO-idebenone and incubated for another $24 \mathrm{~h}$. Fibroblasts treated with $5 \mu \mathrm{M}$ of idebenone served as a positive control, while fibroblasts in complete DMEM without treatment served as a negative control. After incubation, the medium was removed and subjected to total glutathione and GSSG measurement according to the manufacturer's protocol of GSH/GSSG-Glo glutathione assay kit (Promega Corporation, Madison, WI, USA) in a multimode microplate reader (SpectraMax M3, Molecular Devices, San Jose, CA, USA).

\subsection{Hoechst 33258 staining of apoptotic nuclei}

Fibroblasts were plated at a density of $5 \times 10^{3}$ cells per well in black 96-well plates and incubated for $24 \mathrm{~h}$ at $37 \pm 2{ }^{\circ} \mathrm{C}$ in a $5 \% \mathrm{CO}_{2}$-humidified incubator. Then, the medium was replaced with fresh medium containing BSO, BSO-HESAE or BSO-idebenone. Fibroblasts treated with $5 \mu \mathrm{M}$ idebenone served as a positive control, while fibroblasts in complete DMEM without treatment served as a negative control. After $24 \mathrm{~h}$ of incubation, the medium was discarded and loaded with $5 \mu \mathrm{g} / \mathrm{mL}$ Hoechst 33258 in PBS, further incubated in the dark for $10 \mathrm{~min}$ and washed twice with PBS. Images were acquired on Nikon Eclipse Ti-S inverted microscope and Intensilight C-HGFI Precentered Fiber Illuminator (Nikon Corporation, Tokyo, Japan) at $460 \mathrm{~nm}$. Bright-blue fluorescence indicated nuclear apoptosis. ImageJ (National Institutes of Health, 2019) was used to quantify the blue fluorescence intensity in nine randomly chosen microscopic fields (Zhu et al., 2015).

\subsection{Statistical analysis}

Statistical significance was analyzed with independent t-test and one-way ANOVA followed by Tukey's and Dunnett's multiple comparison tests. Differences with $\mathrm{p}$ value less than $0.05(\mathrm{p}<0.05)$ were considered statistically significant.

\section{Results}

\subsection{In vitro antioxidant activities of HESAE}

Table 1 shows the phytochemical contents and in vitro antioxidant activities of HESAE on DPPH and ABTS free radical scavenging, and reducing power assays. The total phenolic and flavonoid contents of HESAE were found to be $7.19 \pm 0.6 \mathrm{mg} \mathrm{GAE} / \mathrm{g}$ and $5.31 \pm 0.3 \mathrm{mg} \mathrm{QE} / \mathrm{g}$, respectively. HESAE exhibited similar levels of total flavonoid content, DPPH and ABTS free radical scavenging activities $(p>0.05)$ as compared to idebenone and possessed significantly higher total phenolic content $(\mathrm{p}<0.05)$ of $7.19 \pm 0.6 \mathrm{mg} \mathrm{GAE} / \mathrm{g}$ and lower $\mathrm{EC}_{50}$ value $(\mathrm{p}<0.05)$ of $58.23 \pm 3.9 \mathrm{mg} / \mathrm{mL}$ in reducing power than idebenone.

\subsection{Effects of BSO on the viability of normal and FRDA fibroblasts}

The vulnerability of normal and FRDA fibroblast to oxidative damage caused by glutathione depletion was investigated through exposure to BSO at varying concentrations ranged from 1.56 to $100 \mathrm{mM}$.

Table 1. Phytochemical contents and antioxidant capacity of HESAE and idebenone. Values were represented as mean \pm SD of triplicates. Means with different alphabets in the same assay were significantly different $(\mathrm{p}<0.05)$.

\begin{tabular}{cccccc}
\hline \multirow{2}{*}{ HESAE / Idebenone } & $\begin{array}{c}\text { Total phenolic content } \\
(\mathrm{mg} \text { GAE/g) }\end{array}$ & $\begin{array}{c}\text { Total flavonoid content } \\
(\mathrm{mg} \text { QE/g) }\end{array}$ & & $\begin{array}{c}\mathrm{DPPH} \\
\text { radical scavenging }\end{array}$ & \multicolumn{2}{c}{$\begin{array}{c}\mathrm{EC}_{50}(\mathrm{mg} / \mathrm{mL}) \\
\text { radical scavenging }\end{array}$} \\
\hline HESAE & $7.19 \pm 0.6^{\mathrm{a}}$ & $5.31 \pm 0.3$ & $13.22 \pm 0.3$ & $14.34 \pm 0.9$ & $58.23 \pm 3.9^{\mathrm{a}}$ \\
Idebenone & $2.38 \pm 0.7^{\mathrm{b}}$ & $4.73 \pm 0.5$ & $16.34 \pm 4.3$ & $17.74 \pm 2.5$ & $120.19 \pm 19.99^{\mathrm{b}}$ \\
\hline
\end{tabular}

HESAE $=$ standardized aqueous extract from fruiting bodies of Hericium erinaceus; $\mathrm{EC}_{50}=$ half-maximal effective concentration; DPPH $=2,2$-diphenyl-1-picrylhydrazyl; $\mathrm{ABTS}=2,2^{\prime}$-azinobis(3-ethylbenzothiazoline-6-sulfonic acid). 
Figure 1 shows morphological features of normal [A(I)-D(I)] and FRDA fibroblasts [A(II)-D(II)] following incubation in BSO for $24 \mathrm{~h}$. At 12.5 and $25 \mathrm{mM}$ BSO, FRDA fibroblast showed distinct alteration with floating debris and loss of spindle shape [C(II)-D(II)]. However, normal fibroblasts retained their typical spindle shape at 6.25 and $12.5 \mathrm{mM}$ BSO [B(I)-C(I)]. Figure $1 \mathrm{E}$ shows the dose-response curve of viability of normal and FRDA fibroblasts following exposure to varying concentrations of BSO for $24 \mathrm{~h}$. All tested concentrations of BSO significantly reduced $(\mathrm{p}<0.05)$ the viability of FRDA fibroblasts. The viability of FRDA fibroblasts decreased gradually as the concentration of BSO increases with a sharp decrease in viability at $12.5 \mathrm{mM}$ BSO. Large-scale death of FRDA fibroblasts was markedly pronounced by challenging the cells with $12.5 \mathrm{mM}$ BSO in which the viability
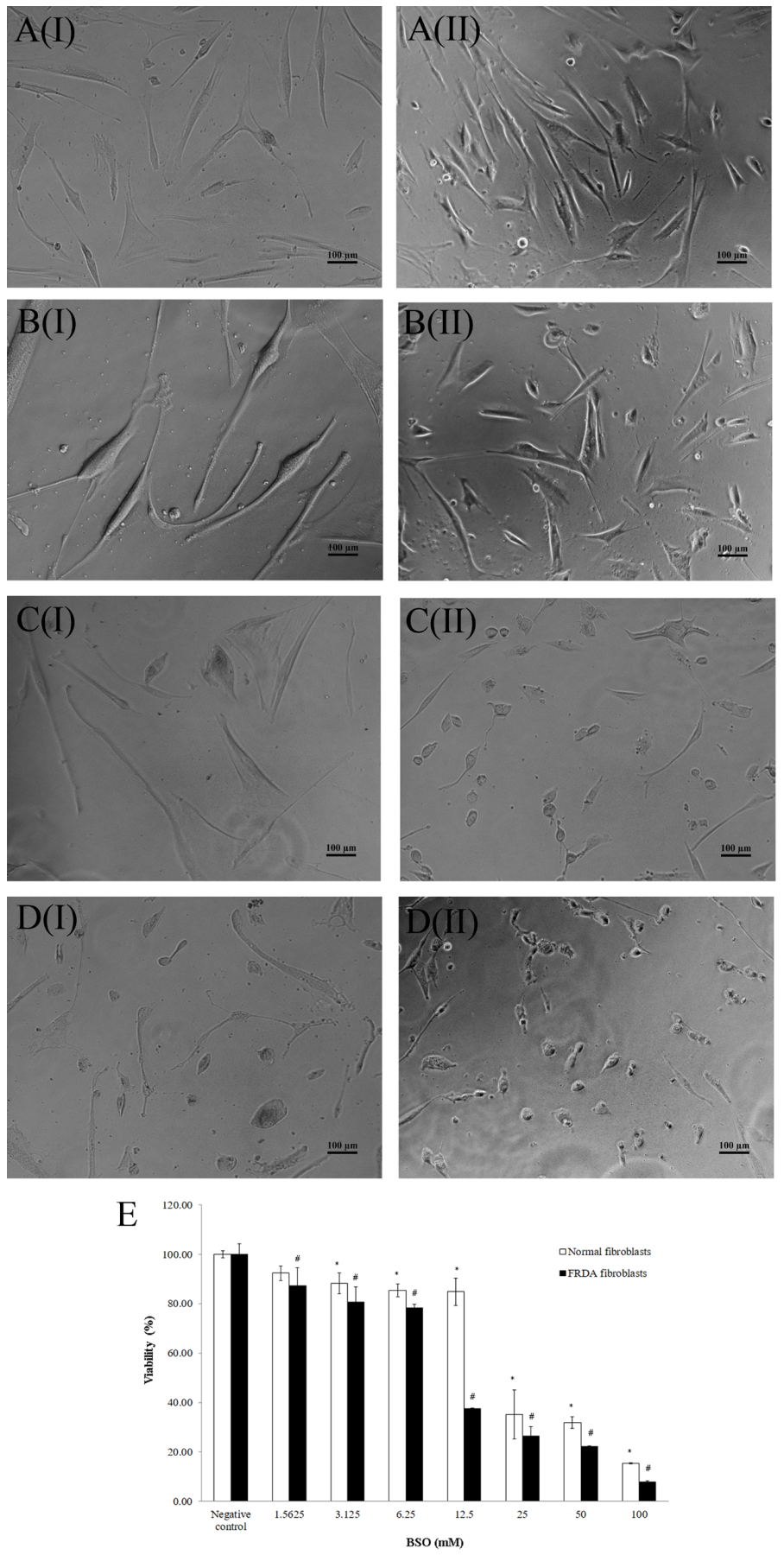

Figure 1. Morphological features of normal and FRDA fibroblasts following incubation with varying concentrations of L-Buthionine sulfoximine (BSO) for 24h. A(I) to D(I) Normal fibroblasts. A(II) to D(II) FRDA fibroblasts. A(I) and A(II) Normal and FRDA fibroblasts in negative control group; B(I) and B(II) Normal and FRDA fibroblasts exposed to $6.25 \mathrm{mM} \mathrm{BSO}$; C(I) and C(II) Normal and FRDA fibroblasts exposed to $12.5 \mathrm{mM}$ BSO; D(I) and D(II) Normal and FRDA fibroblasts exposed to $25 \mathrm{mM}$ BSO. Scale bar $=100 \mu \mathrm{m}$; (E) Percentage of viability of normal and FRDA fibroblasts following incubation with varying concentrations of BSO for $24 \mathrm{~h}$. Asterisks $\left(^{*}\right)$ and hash signs (\#) indicate significant differences $(\mathrm{p}<0.05)$ in viability for different groups of normal and FRDA fibroblasts, respectively, compared to the negative control group. 
was reduced to $37.47 \pm 0.23 \%$. On the other hand, $12.5 \mathrm{mM} \mathrm{BSO}$ produced mild cytotoxicity effects in normal fibroblasts.

As $12.5 \mathrm{mM}$ BSO produced more than $50 \%$ reduction $(\mathrm{p}<0.05)$ in the viability of FRDA fibroblasts, therefore the concentration was selected in the subsequent assays to induce oxidative damage in FRDA fibroblasts.

\subsection{Effects of HESAE on the viability of normal and FRDA fibroblasts}

Prior to the investigation of cytoprotective activities, the effects of HESAE to the viability of normal and FRDA fibroblasts was assessed by MTT assay to exclude any possibilities of cytotoxicity effects of the extract. Figure 2 shows that HESAE in the range of 1 to $32 \mathrm{mg} / \mathrm{mL}$ exerted neither inhibition nor increase in the viability of fibroblasts, and therefore was selected for $\mathrm{LDH}$ viability assay to study its protective effects against $12.5 \mathrm{mM} \mathrm{BSO}$-induced cytotoxicity in FRDA fibroblasts. Further, the 50\% inhibitory concentration of HESAE on normal and FRDA fibroblasts was $52.26 \mathrm{mg} / \mathrm{mL}$ and $46.77 \mathrm{mg} / \mathrm{mL}$, respectively.

\subsection{Effects of HESAE on the viability of BSO-treated FRDA fibroblasts determined by quantification of $L D H$}

The protective effects of HESAE against BSO-induced cytotoxicity in FRDA fibroblasts was investigated by co-incubating varying concentrations of HESAE with $12.5 \mathrm{mM} \mathrm{BSO}$. As shown in Figure 3, the viability of FRDA fibroblasts was reduced from $100.00 \pm 0.8 \%$ to $52.51 \pm 13.92 \%$ by $12.5 \mathrm{mM} \mathrm{BSO}(\mathrm{p}<0.05)$. However, co-incubation with $32 \mathrm{mg} / \mathrm{mL}$ HESAE significantly increased the viability of FRDA fibroblasts to $85.35 \pm 3.4 \%(\mathrm{p}<0.05)$ that was 2 -fold higher compared to $5 \mu \mathrm{M}$ idebenone at $40.09 \pm 0.41 \%(\mathrm{p}<0.05)$.

Mechanisms of cell death and survival were further examined by quantification of glutathione content and induction of apoptosis. As $32 \mathrm{mg} / \mathrm{mL}$ HESAE promoted highest percentage of viability of BSO-induced oxidative damage in FRDA fibroblasts, the concentration was selected for subsequent assays.

\subsection{Effects of HESAE on the ratio of GSH/GSSG in BSO-treated FRDA fibroblast}

This reduction in the GSH/GSSG ratio indicates redox imbalance and increase in oxidants due to neurodegenerative diseases. The GSH/GSSG ratio was determined in FRDA fibroblasts following co-incubation of $12.5 \mathrm{mM}$ BSO with $32 \mathrm{mg} / \mathrm{mL}$ HESAE for $24 \mathrm{~h}$. As shown in Figure 4, BSO significantly depleted the GSH/GSSG ratio up to 50-fold compared to negative control $(\mathrm{p}<0.05)$, and HESAE appeared to increase the GSH/GSSG ratio up to 17 -fold and 80 -fold, compared to BSO and BSO-idebenone groups, respectively $(\mathrm{p}<0.05)$. The data show that HESAE prevented BSO from depleting intracellular GSH/GSSG ratio or induced glutathione synthesis in vitro.

\subsection{Effects of HESAE on nuclear apoptosis in BSO-treated FRDA fibroblasts}

As shown in Figure 5A-D, nuclei of untreated FRDA fibroblasts exhibited homogeneously dim-blue and round shape structure after labeling with Hoechst 33258. The introduction of $12.5 \mathrm{mM}$ BSO

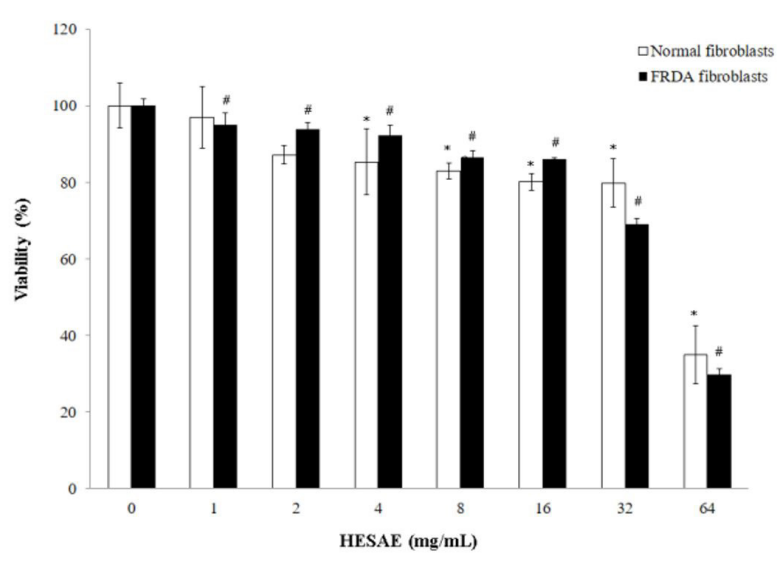

Figure 2. Effects of HESAE on viability of normal and FRDA fibroblasts detected by MTT assay following incubation with varying concentrations of HESAE for $24 \mathrm{~h}$. Asterisks $\left({ }^{*}\right)$ and hash signs (\#) indicate significant differences $(\mathrm{p}<0.05)$ in viability for different groups of normal and FRDA fibroblasts, respectively, compared to the negative control group.

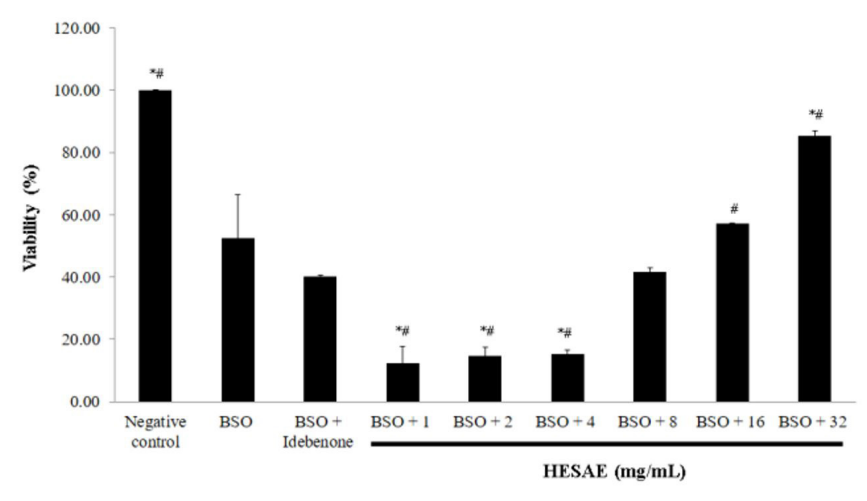

Figure 3. Effects of HESAE on viability of FRDA fibroblasts detected by LDH assay following co-incubation of $12.5 \mathrm{mM}$ BSO and varying concentrations of HESAE for $24 \mathrm{~h}$. Asterisks $\left(^{*}\right)$ and hash signs (\#) indicate significant differences $(\mathrm{p}<0.05)$ in viability for different groups compared to the $\mathrm{BSO}$-treated group and $\mathrm{BSO}$-idebenone group, respectively.

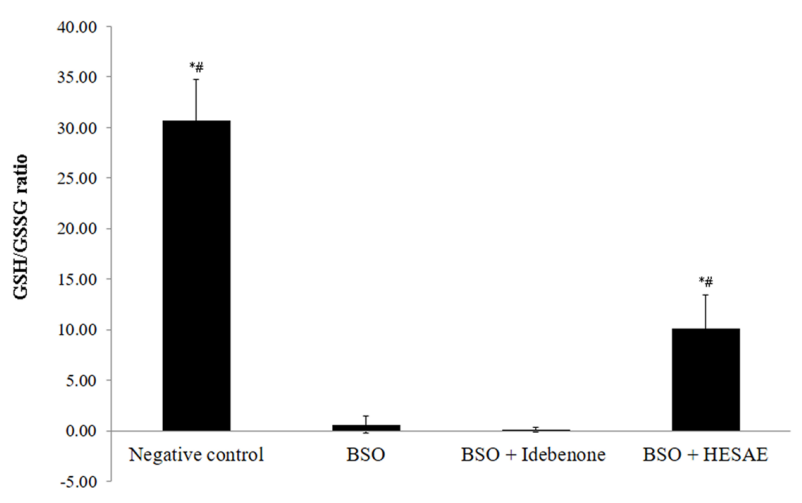

Figure 4. Effects of HESAE on GSH/GSSG ratio in BSO-treated FRDA fibroblasts following co-incubation of $12.5 \mathrm{mM}$ BSO and $32 \mathrm{mg} / \mathrm{mL}$ HESAE for $24 \mathrm{~h}$. Asterisks $\left(^{*}\right)$ and hash signs (\#) indicate significant differences $(\mathrm{p}<0.05)$ in GSH/GSSG ratio for different groups compared to the $\mathrm{BSO}$-treated group and $\mathrm{BSO}$-idebenone group, respectively. 
into FRDA fibroblasts causes induction of apoptosis indicated by bright-blue fluorescence. However, co-incubation with $5 \mu \mathrm{M}$ idebenone or $32 \mathrm{mg} / \mathrm{mL}$ HESAE reduced the fluorescence intensity. Figure 5E shows that BSO significantly increased the fluorescence intensity from $100.00 \pm 8.73 \%$ to $121.74 \pm 2.04 \%$ ( $\mathrm{p}<0.05)$. The intensity was reduced to $98.74 \pm 4.23 \%(\mathrm{p}<0.05)$ by co-incubation with $32 \mathrm{mg} / \mathrm{mL}$ HESAE. On the other hand, the intensity of $153.41 \pm 2.08 \%$ was obtained by co-incubation with $5 \mu \mathrm{M}$ idebenone.

\section{Discussion}

Natural products have been traditionally accepted as remedies due to popular belief that they present minor adverse effects. There is currently no reports of natural product that possesses protective effects in an in vitro model of FRDA.
This circumstance stimulated our interest to explore HESAE and to probe the mechanism underlying the protective effects in FRDA.

Phenolic acids that possess hydroxyl groups are the major bioactive components in medicinal mushrooms and are also associated with their antioxidant properties (Ferreira et al., 2009; Heleno et al., 2012). The total phenolic content of HESAE obtained from this study was higher compared to that of methanolic extracts of fresh fruiting bodies $(0.26 \mathrm{mg}$ GAE/g extract), oven-dried fruiting bodies (2.37 mg GAE/g extract), and freeze-dried fruiting bodies ( $0.78 \mathrm{mg}$ GAE/g extract) of H. erinaceus (Wong et al., 2009).

In a study by Hou et al. (2015), a purified oligosaccharide isolated from $H$. erinaceus water extract (HEO-A) possessed DPPH
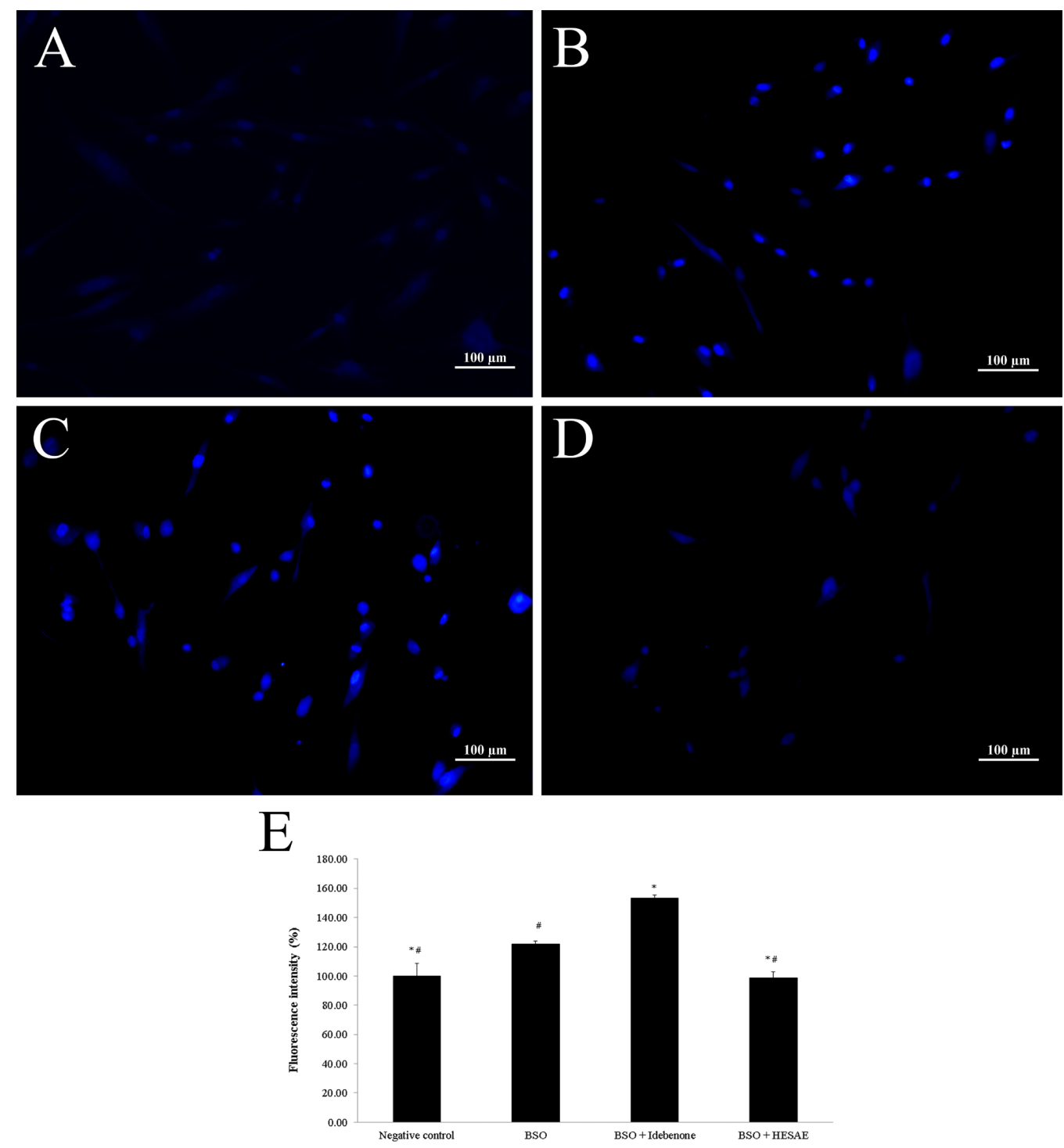

Figure 5. FRDA fibroblasts stained as blue fluorescence with Hoechst 33258 for the detection of apoptotic nuclei after $24 \mathrm{~h}$. (A) FRDA fibroblasts in negative control group; (B) FRDA fibroblasts exposed to $12.5 \mathrm{mM}$ BSO; (C) FRDA fibroblasts following co-incubation of $12.5 \mathrm{mM}$ BSO and $5 \mu \mathrm{M}$ idebenone; (D) FRDA fibroblasts following co-incubation of $12.5 \mathrm{mM} \mathrm{BSO}$ and $32 \mathrm{mg} / \mathrm{mL}$ HESAE. Scale bar $=100 \mu \mathrm{m}$; (E) Intensity of Hoechst 33258 blue fluorescence in the FRDA fibroblasts. Asterisks $\left(^{*}\right)$ and hash signs $(\#)$ indicate significant differences $(p<0.05)$ in fluorescence intensity for different groups compared to the BSO-treated group and BSO-idebenone group, respectively. 
radical scavenging activity at $\mathrm{EC}_{50}$ value of $\sim 12.5 \mathrm{mg} / \mathrm{mL}$ which was almost similar compared to HESAE at $13.22 \pm 0.3 \mathrm{mg} / \mathrm{mL}$ and an $\mathrm{EC}_{50}$ value of $0.93 \mathrm{mg} / \mathrm{mL}$ in ABTS radical scavenging activity which was 15 -fold higher compared to $14.34 \pm 0.9 \mathrm{mg} / \mathrm{mL}$ exhibited by HESAE. Nevertheless, it has been demonstrated that a purified compound may not necessary possess antioxidant function compared to its extract counterpart. Ethanol and water extracts of $H$. erinaceus showed more potent reducing power and free radical scavenging activities compared to low polarity solvents, indicating that antioxidant compounds are often concentrated in more polar solvents (Jiang et al., 2016). HESAE was prepared by high polarity aqueous extraction of fresh fruiting bodies to ensure total extractable phenolic, flavonoid and antioxidant contents (Mau et al., 2002; Wong et al., 2009).

Dermal fibroblasts from FRDA patients is a widely accepted in vitro model for studying FRDA (Jauslin et al., 2002, 2003). The FRDA fibroblasts are homozygous for trinucleotide GAA expansion in the frataxin gene with alleles of approximately 541 and 420 repeats. Functional frataxin is crucial in the production of Fe-S cluster containing proteins (Richardson et al., 2012).

Oxidative damage has been shown to be a key element in the pathogenesis of FRDA. Interestingly, we found that FRDA fibroblasts were sensitive to BSO-induced oxidative damage at $12.5 \mathrm{mM}$ compared to normal fibroblasts that required higher concentrations of BSO to obtain a comparable toxicity. Glutathione is a redox-active molecule existing in the forms of thiol (GSH) and disulphide (GSSG). Reduced level of glutathione causes irreversible inhibition of $\gamma$-glutamylcysteine synthetase, the rate-limiting enzyme in the biosynthesis of GSH after exposure to BSO. A chronic deficit in the GSH/GSSG ratio is an indicator of oxidative stress and renders mitochondria more vulnerable to oxidative damage/dysfunction during aging, multiple chronic diseases and cataracts (Lodi et al., 2006). Remarkably, our results demonstrated that HESAE increased the ratio compared to BSO or co-incubation with idebenone. Nevertheless, our present findings are in contrast to Richardson et al. (2012) who reported that estrogen-like compounds failed to prevent BSO from causing GSH depletion or to increase the expression of GSH.

Figure 6 demonstrates the mechanisms by which BSO promotes apoptosis in FRDA fibroblasts and protective effects of HESAE against the oxidative damage. As FRDA fibroblasts are lacking in frataxin, the cells are extremely sensitive to BSO-induced oxidative stress compared to normal fibroblasts. Frataxin has been shown to be influential in the production of Fe-S cluster containing proteins (Richardson et al., 2012). We postulated that synthesis of glutathione in FRDA fibroblasts was greatly diminished following incubation in $\mathrm{BSO}$ as evidenced by extreme reduction in GSH/GSSG ratio up to 50-fold. Disruption in the redox state of FRDA fibroblasts resulted in an increase of cytosolic LDH causing a $48 \%$ reduction in viability that leads to $22 \%$ increase in apoptosis compared to negative control. Viability of FRDA fibroblasts was inhibited by more than half with prominent alteration in the morphology. However, HESAE could attenuate the cytotoxicity effects through the prevention of glutathione depletion, decreasing the level of LDH that reflects the integrity of plasma membrane and anti-apoptotic activity. The elevation in serum LDH level is a reliable biomarker of FRDA. In a case report by Krongrad \& Joos (1972), a 7-year-old Caucasian girl with FRDA and myocardial infarction had persistent elevation of cardiac specific LDH isoenzyme 5.

Antioxidant therapy with idebenone is currently the only treatment option resulting in an improvement of hypertrophic cardiomyopathy (Jauslin et al., 2007). In our study, idebenone did not protect BSO-induced oxidative insult to FRDA fibroblast as measured by apoptotic cell death and release of $\mathrm{LDH}$ from damaged cells owing to membrane disintegration. Several studies have also reported conflicting results of idebenone in cardiovascular clinical trials of FRDA (Kearney et al., 2016). FRDA comprises both neurological symptoms and cardiac hypertrophy. Irreversible destruction of neurons and poor antioxidant effect of idebenone in the neurons may contribute to the differential effect observed in FRDA patients with cardiac

\section{Friedreich's ataxia}

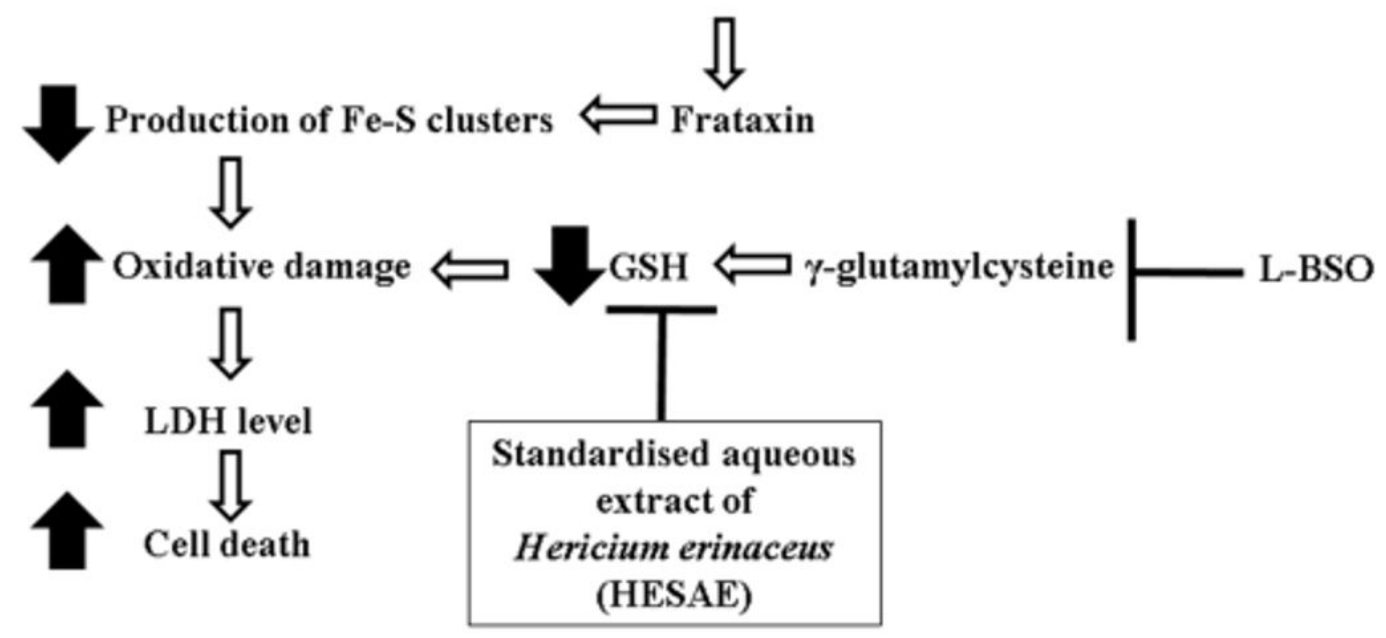

Figure 6. Protective mechanism of HESAE in BSO-exposed FRDA dermal fibroblasts. 
hypertrophy and neurological symptoms. Idebenone did not have noticeable effects on ataxia (Rustin, 2003).

Ebselen, a glutathione peroxidase mimetic (Jauslin et al., 2002) and a novel synthetic antioxidant (Fe-Aox29) combining the active groups from idebenone and vitamin $\mathrm{E}$ (Jauslin et al., 2007) are more potent in preventing BSO-mediated cell death in FRDA fibroblasts compared to either idebenone or vitamin E alone. Similarly, 17 $\beta$-estradiol and estrogen-like compounds (Richardson et al., 2011), estrogen receptor $\beta$ agonist of $\mathrm{R}$ - and S-forms of equol biphenolic compounds (Richardson \& Simpkins, 2012) and phenolic estrogens (Richardson et al., 2012) were able to prevent cell death in FRDA fibroblasts. Compounds with increased numbers of phenolic rings showed an increased protective effect. The phenolic or hydroxyl group-containing estrogens were able to prevent lipid peroxidation and mitochondrial membrane potential collapse, maintain the level of ATP and aconitase, and increase oxidative phosphorylation in FRDA fibroblasts (Richardson et al., 2012). Based on our phytochemical findings, we have demonstrated a potential protective effects of HESAE against oxidative damage in FRDA fibroblast that was attributed to its high phenolic content.

\section{Conclusion}

In this study, a standardized aqueous extract from fresh fruiting bodies of $H$. erinaceus was found to be beneficial in attenuating BSO-mediated oxidative damage in FRDA fibroblasts. Our data support the hypothesis that antioxidative capability of the extract could be responsible for prevention of oxidative damage. It remains to be determined if the use of pure antioxidant molecules isolated from the extract can further amplify defences against the oxidative insult.

\section{Acknowledgements}

This work was funded by Fundamental Research Grant Scheme FP011-2016 from the Ministry of Higher Education Malaysia and University of Malaya Faculty of Medicine Research Grant GPF003C-2019. We would like to thank Ganofarm R\&D Private Limited, Tanjung Sepat, Selangor Darul Ehsan, Malaysia for providing the mushroom sample.

\section{References}

Brand-Williams, W., Cuvelier, M. E., \& Berset, C. (1995). Use of a free radical method to evaluate antioxidant activity. LebensmittelWissenschaft + Technologie, 28(1), 25-30. http://dx.doi.org/10.1016/ S0023-6438(95)80008-5.

Delatycki, M. B., \& Corben, L. A. (2012). Clinical features of Friedreich ataxia. Journal of Child Neurology, 27(9), 1133-1137. http://dx.doi. org/10.1177/0883073812448230. PMid:22752493.

Ferreira, I. C., Barros, L., \& Abreu, R. (2009). Antioxidants in wild mushrooms. Current Medicinal Chemistry, 16(12), 1543-1560. http://dx.doi.org/10.2174/092986709787909587. PMid:19355906.

Friedman, M. (2015). Chemistry, nutrition, and health-promoting properties of Hericium erinaceus (lion's mane) mushroom fruiting bodies and mycelia and their bioactive compounds. Journal of Agricultural and Food Chemistry, 63(32), 7108-7123. http://dx.doi. org/10.1021/acs.jafc.5b02914. PMid:26244378.
Heleno, S. A., Barros, L., Martins, A., Queiroz, M. J. R., Santos-Buelga, C., \& Ferreira, I. C. (2012). Phenolic, polysaccharidic, and lipidic fractions of mushrooms from Northeastern Portugal: chemical compounds with antioxidant properties. Journal of Agricultural and Food Chemistry, 60(18), 4634-4640. http://dx.doi.org/10.1021/ jf300739m. PMid:22515547.

Hou, Y., Ding, X., \& Hou, W. (2015). Composition and antioxidant activity of water-soluble oligosaccharides from Hericium erinaceus. Molecular Medicine Reports, 11(5), 3794-3799. http://dx.doi. org/10.3892/mmr.2014.3121. PMid:25529054.

Inanaga, K. (2014). Marked improvement of neurocognitive impairment after treatment with compounds from Hericium erinaceum: a case study of recurrent depressive disorder. Personalized Medicine Universe, 3, 46-48. http://dx.doi.org/10.1016/j.pmu.2014.02.004.

Jauslin, M. L., Meier, T., Smith, R. A., \& Murphy, M. P. (2003). Mitochondria-targeted antioxidants protect Friedreich ataxia fibroblasts from endogenous oxidative stress more effectively than untargeted antioxidants. The FASEB Journal, 17(13), 1972-1974. http://dx.doi.org/10.1096/fj.03-0240fje. PMid:12923074.

Jauslin, M. L., Vertuani, S., Durini, E., Buzzoni, L., Ciliberti, N., Verdecchia, S., Palozza, P., Meier, T., \& Manfredini, S. (2007). Protective effects of Fe-Aox29, a novel antioxidant derived from a molecular combination of idebenone and vitamin $\mathrm{E}$, in immortalized fibroblasts and fibroblasts from patients with Friedreich ataxia. Molecular and Cellular Biochemistry, 302(1-2), 79-85. http://dx.doi. org/10.1007/s11010-007-9429-2. PMid:17476463.

Jauslin, M. L., Wirth, T., Meier, T., \& Schoumacher, F. (2002). A cellular model for Friedreich ataxia reveals small-molecule glutathione peroxidase mimetics as novel treatment strategy. Human Molecular Genetics, 11(24), 3055-3063. http://dx.doi.org/10.1093/hmg/11.24.3055. PMid:12417527.

Jiang, S., Wang, Y., \& Zhang, X. (2016). Comparative studies on extracts from Hericium erinaceus by different polarity reagents to gain higher antioxidant activities. Experimental and Therapeutic Medicine, 12(1), 513-517. http://dx.doi.org/10.3892/etm.2016.3279. PMid:27347087.

Kearney, M., Orrell, R. W., Fahey, M., Brassington, R., \& Pandolfo, M. (2016). Pharmacological treatments for Friedreich ataxia. Cochrane Database of Systematic Reviews, (8), CD007791. PMid:27572719.

Koeppen, A. H. (2013). Nikolaus Friedreich and degenerative atrophy of the dorsal columns of the spinal cord. Journal of Neurochemistry, 126(Suppl. 1), 4-10. http://dx.doi.org/10.1111/jnc.12218. PMid:23859337.

Krongrad, E., \& Joos, H. A. (1972). Friedreich's ataxia in childhood. Case report with possible myocardial infarction, cerebrovascular thromboembolization, and persistent elevation of cardiac specific LDH. Chest, 61(7), 644-648. http://dx.doi.org/10.1378/chest.61.7.644. PMid:5031758.

Lodi, R., Tonon, C., Calabrese, V., \& Schapira, A. H. (2006). Friedreich's ataxia: from disease mechanisms to therapeutic interventions. Antioxidants \& Redox Signalling, 8(3-4), 438-443. http://dx.doi. org/10.1089/ars.2006.8.438. PMid:16677089.

Mau, J. L., Lin, H. C., \& Chen, C. C. (2002). Antioxidant properties of several medicinal mushrooms. Journal of Agricultural and Food Chemistry, 50(21), 6072-6077. http://dx.doi.org/10.1021/jf0201273. PMid:12358482.

Miller, N. J., Rice-Evans, C., Davies, M. J., Gopinathan, V., \& Milner, A. (1993). A novel method for measuring antioxidant capacity and its application to monitoring the antioxidant status in premature neonates. Clinical Science, 84(4), 407-412. http://dx.doi.org/10.1042/ cs0840407. PMid:8482045.

Nagano, M., Shimizu, K., Kondo, R., Hayashi, C., Sato, D., Kitagawa, K., \& Ohnuki, K. (2010). Reduction of depression and anxiety by 4 
weeks Hericium erinaceus intake. Biomedical Research, 31(4), 231237. http://dx.doi.org/10.2220/biomedres.31.231. PMid:20834180.

National Institutes of Health - NIH. (2019). ImageJ. Bethesda. Retrieved from http://imagej.nih.gov/ij/

Okamura, H., Anno, N., Tsuda, A., Inokuchi, T., Uchimura, N., \& Inanaga, K. (2015). The effects of Hericium erinaceus (Amyloban ${ }^{\circledR}$ 3399) on sleep quality and subjective well-being among female undergraduate students: a pilot study. Personalized Medicine Universe, 4, 76-78. http://dx.doi.org/10.1016/j.pmu.2015.03.006.

Oyaizu, M. (1986). Studies on product of browning reaction prepared from glucose amine. Japan Journal of Nutrition, 44(6), 307-315. http://dx.doi.org/10.5264/eiyogakuzashi.44.307.

Pang, J. R., Goh, V. M. J., Tan, C. Y., Phang, S. M., Wong, K. H., \& Yow, Y. Y. (2018). Neuritogenic and in vitro antioxidant activities of Malaysian Gracilaria manilaensis Yamamoto \& Trono. Journal of Applied Phycology, 30(6), 3253-3260. http://dx.doi.org/10.1007/ s10811-018-1438-x.

Pękal, A., \& Pyrzynska, K. (2014). Evaluation of aluminium complexation reaction for flavonoid content assay. Food Analytical Methods, 7(9), 1776-1782. http://dx.doi.org/10.1007/s12161-014-9814-x.

Reliene, R., \& Schiestl, R. H. (2006). Glutathione depletion by buthionine sulfoximine induces DNA deletions in mice. Carcinogenesis, 27(2), 240-244. http://dx.doi.org/10.1093/carcin/bgi222. PMid:16162646.

Richardson, T. E., \& Simpkins, J. W. (2012). R-and S-Equol have equivalent cytoprotective effects in Friedreich's ataxia. BMC Pharmacology \& Toxicology, 13(1), 12. http://dx.doi.org/10.1186/2050-6511-13-12. PMid:23088310.

Richardson, T. E., Yang, S. H., Wen, Y., \& Simpkins, J. W. (2011). Estrogen protection in Friedreich's ataxia skin fibroblasts. Endocrinology, 152(7), 2742-2749. http://dx.doi.org/10.1210/en.2011-0184. PMid:21540287.

Richardson, T. E., Yu, A. E., Wen, Y., Yang, S. H., \& Simpkins, J. W. (2012). Estrogen prevents oxidative damage to the mitochondria in Friedreich's ataxia skin fibroblasts. PLoS One, 7(4), e34600. http:// dx.doi.org/10.1371/journal.pone.0034600. PMid:22509330.
Rustin, P. (2003). The use of antioxidants in Friedreich's ataxia treatment. Expert Opinion on Investigational Drugs, 12(4), 569-575. http:// dx.doi.org/10.1517/13543784.12.4.569. PMid:12665413.

Singleton, V. L., Orthofer, R., \& Lamuela-Raventos, R. M. (1999). Analysis of total phenols and other oxidation substrates and antioxidants by means of Folin-Ciocalteu reagent. Methods in Enzymology, 299, 152-178. http://dx.doi.org/10.1016/S0076-6879(99)99017-1.

Wong, K. H., Kanagasabapathy, G., Bakar, R., Phan, C. W., \& Sabaratnam, V. (2015). Restoration of sensory dysfunction following peripheral nerve injury by the polysaccharide from culinary and medicinal mushroom, Hericium erinaceus (Bull.: Fr.) Pers. through its neuroregenerative action. Food Science and Technology, 35(4), 712721. http://dx.doi.org/10.1590/1678-457X.6838.

Wong, K. H., Naidu, M., David, P., Abdulla, M. A., Abdullah, N., Kuppusamy, U. R., \& Sabaratnam, V. (2011). Peripheral nerve regeneration following crush injury to rat peroneal nerve by aqueous extract of medicinal mushroom Hericium erinaceus (Bull.: Fr) Pers. (Aphyllophoromycetideae). Evidence-Based Complementary and Alternative Medicine, 2011, 580752. http://dx.doi.org/10.1093/ecam/ neq062. PMid:21941586.

Wong, K. H., Sabaratnam, V., Abdullah, N., Kuppusamy, U. R., \& Naidu, M. (2009). Effects of cultivation techniques and processing on antimicrobial and antioxidant activities of Hericium erinaceus (Bull.: Fr.) Pers. extracts. Food Technology and Biotechnology, 47(1), 47-55.

Wong, K.-H., Vikineswary, S., Abdullah, N., Naidu, M., \& Keynes, R. (2007). Activity of aqueous extracts of lion's mane mushroom Hericium erinaceus (Bull.: Fr.) Pers. (Aphyllophoromycetideae) on the neural cell line NG108-15. International Journal of Medicinal Mushrooms, 9(1), 57-65. http://dx.doi.org/10.1615/IntJMedMushr. v9.i1.70.

Zhu, H., Tang, Y., Zhang, X., Jiang, X., Wang, Y., Gan, Y. U., \& Yang, J. (2015). Downregulation of UPK1A suppresses proliferation and enhances apoptosis of bladder transitional cell carcinoma cells. Medical Oncology, 32(3), 84. http://dx.doi.org/10.1007/s12032-0150541-y. PMid:25701463. 